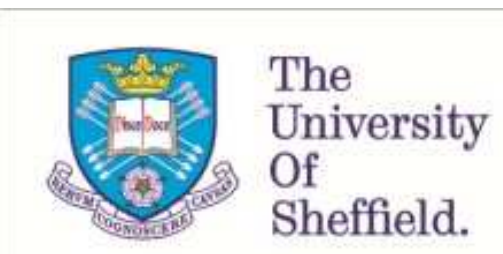

This is a repository copy of Towards a framework for tactile perception in social robotics.

White Rose Research Online URL for this paper:

http://eprints.whiterose.ac.uk/155166/

Version: Accepted Version

\title{
Proceedings Paper:
}

Barron-Gonzalez, H., Lepora, N.F., Martinez-Hernandez, U. et al. (2 more authors) (2012) Towards a framework for tactile perception in social robotics. In: Prescott, T.J., Lepora, N.F., Mura, A. and Verschure, P.F.M.J., (eds.) Biomimetic and Biohybrid Systems. Living Machines 2012. First International Conference, Living Machines 2012, 09-12 Jul 2012, Barcelona, Spain. Lecture Notes in Computer Science (7375). Springer , pp. 335-336. ISBN 9783642315244

https://doi.org/10.1007/978-3-642-31525-1_30

This is a post-peer-review, pre-copyedit version of an article published in Lecture Notes in Computer Science. The final authenticated version is available online at:

http://dx.doi.org/10.1007/978-3-642-31525-1_30

\section{Reuse}

Items deposited in White Rose Research Online are protected by copyright, with all rights reserved unless indicated otherwise. They may be downloaded and/or printed for private study, or other acts as permitted by national copyright laws. The publisher or other rights holders may allow further reproduction and re-use of the full text version. This is indicated by the licence information on the White Rose Research Online record for the item.

\section{Takedown}

If you consider content in White Rose Research Online to be in breach of UK law, please notify us by emailing eprints@whiterose.ac.uk including the URL of the record and the reason for the withdrawal request. 


\title{
Towards a Framework for Tactile Perception in Social Robotics
}

\author{
Hector Barron-Gonzalez, Nathan F. Lepora, Uriel Martinez-Hernandez, \\ Mat Evans, and Tony J. Prescott \\ Department of Psychology, University of Sheffield, Western Bank, S10 2TP, UK \\ \{hector.barron, n.lepora, uriel.martinez, m.evans, \\ t.j.prescott\}@sheffield.ac.uk
}

The first step for building reliable humanoid systems is to provide them with perceptual mechanisms that have human attributes, such as the skill development, social interaction, environmental embodiment and sensorial integration. Despite tactile perception being one of the most important elements for human interaction with the world, its implementation within artificial systems has been tardy, principally because it requires a complete integration with the motor systems and an environmental coupling to extract comprehensible information [1]. Thus, this work aims to generate a platform based on haptic information, allowing humanoids to perceive and represent surrounding objects using concepts fully grounded in the sensorial data.

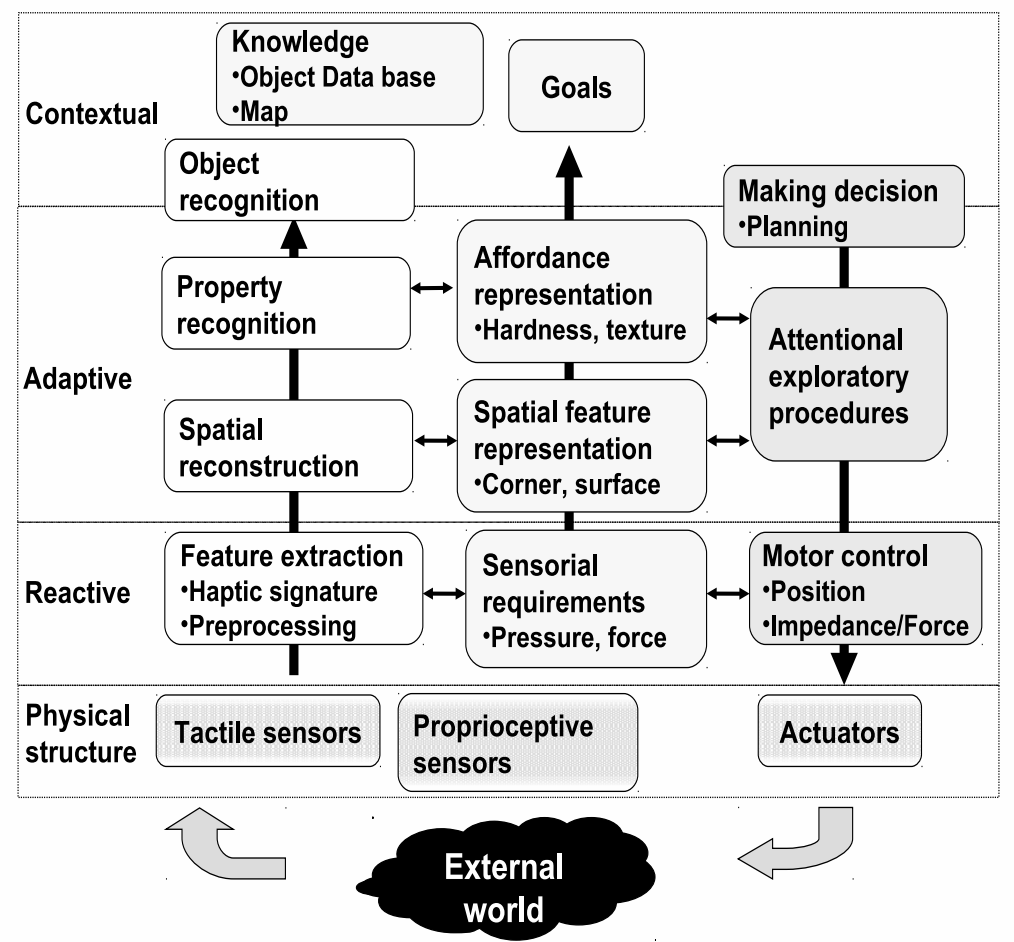

Fig. 1. Functional blocks of the tactile framework

The proposed architecture, inspired in the Distributed Architecture Control [2], has a modular design defined by three levels of processing that represent 
degrees of cognition (see Fig. (1). Such an architecture is not only targeted to build up the perceptual basis for social robotics, but also to explore neuropsychological models for understanding the development of human haptic skills.

The lowest layer is composed of proprioceptive sensors, tactile sensors and actuators. The respective biological elements in the human being are muscles and the mechanoreceptors located in tendons and skin. A higher level of the framework provides enactive mechanisms to extract significant haptic information and control the hand. Engineering advances related to the function of the cerebellum could provide a substantial contribution to haptic manipulation, for example by representing forward and inverse models of the interaction of the manipulator with its environment for optimal control [3].

The second level provides a type of processing that is more representational, such as the physical characteristics of the objects being identified. The implementation of this layer requires that the haptic signatures are grouped in spatiotemporal patterns, generating a hierarchical representation of the object [4]. For instance, probabilistic approaches have been efficiently used to reconstruct the spatial configuration while performing object manipulation [5]. On the other hand, qualitative information is linked to the action that objects afford. Thus, we propose that the appropriate actions should be learnt as new grounded tactile concepts are formed. Such exploratory procedures, driven by attention, provide a mechanism for active perception, as implemented in Bayesian approaches to cortical modelling [6]. Making decisions, recognizing objects and long-term memory are the executive functions for the haptic perception that comprises the highest level of the framework.

We conclude that this framework, designed with an engineering methodology, can in principle be mapped onto a cortical implementation. Furthermore, we propose that the framework be implemented using development cycle that allows the gradual improvement of haptic capabilities in androids, until potentially reaching or exceeding those of humans.

Acknowledgments. This work was supported by FP7 grants EFAA (ICT248986) and the Mexican government under CONACYT.

\section{References}

1. Lederman, S.J., Klatzky, R.L.: Haptic perception: A tutorial. Attention, Perception and Psychophysics 71(7), 1439-1459 (2009)

2. Verschure, P.F.M.J., Voegtlin, T., Douglas, R.J.: Environmentally mediated synergy between perception and behaviour in mobile robots. Nature 425, 620-624 (2003)

3. Wolpert, D.M., Kawato, M.: Multiple paired forward and inverse models for motor control. Neural Networks 11(7-8), 1317-1329 (1998)

4. Hawkins, J., George, D., Niemasik, J.: Sequence memory for prediction, inference and behaviour. Philosophical Transactions of the Royal Society B: Biological Sciences 364(1521), 1203-1209 (2009)

5. Thrun, S., Burgard, W., Fox, D.: Probabilistic robotics. MIT Press (2006)

6. Friston, K.: The free-energy principle. A unified brain theory. Nat. Rev. Neurosci. 11(2), 127-138 (2010) 